\title{
The Characteristics of Chinese Painting and Its Contemporary Heritage
}

\author{
Mingyong Bao \\ Jiangxi Institute of Fashion Technology, Jiangxi, Nanchang, 330201 \\ 1946284603@qq.com
}

Keywords: Chinese painting; brush and ink; characteristics; inheritance

\begin{abstract}
The reason why the brush and ink problem has become the old problem of Chinese painting research is that it is the most fundamental and most central issue of Chinese painting. It is not only the most important expression language of Chinese painting, but also the bearing body of the artist's aesthetic taste and painting concept, as well as the basic measure of analyzing works and criticizing painters. The development of brush and ink in the development of the time, in different historical periods and different stages has significant differences, and continues to improve and mature. This paper first introduced the meaning of Chinese brush and ink, and further elaborated its characteristics. Finally, it discussed the inheritance and innovation of Chinese brush and ink.
\end{abstract}

\section{Introduction}

With its unique form of expression and vivid cultural background, Chinese painting has attracted countless people's admiration. The reason why Chinese painting has such a great charm lies in its performance. Those who draw Chinese paintings must start with ink and ink before they can explore the essence of the painting. The essence of Chinese painting is ink. Looking at the legacy of Chinese history and culture, most of them have the beauty of the soul created by ink and brush. Moreover, among the painting theories of the past to the present, there is also a lot of importance in reminding the pen and ink. For example, Wang Wei, a famous painter in the Tang Dynasty, proposed in the book "The Landscape of the Mountains and Waters": "Ink painting is the most popular among the paintings." In the "Landscape Theory," it is mentioned that "the meaning of the painting is first." The painter of the Song Dynasty, Han Xuan, described the application of the pen and ink method in the Complete Works of Shanshui Pure. Therefore, we can see the special artistry of Chinese brush and ink. The brush and ink give Chinese painting a special creative idea, which explains the significance of brush painting in Chinese painting.

\section{The Meaning of Chinese Brush and Ink}

Chinese brush ink is widely used as the basic method of using Chinese ink and brush in Chinese painting. The pen and ink were highly valued by artists of the dynasties. So, what is the ink? Brush and ink is the material used to express and complete the picture. At the same time, with the development of Chinese traditional culture, in the long process of human painting, it has gradually become an art technique used to express a language tool for Chinese painting. The brushwork of Chinese painting is an integrated and inseparable whole. The two cannot exist independently. Only the use of pens and ink can play its role. As the old saying goes, "Ink is a pen and the ink is the elite." "The ink in the pen is ingenious, and the ink is used in the author." The performance of the painting is not purely "with ink, no pen" or "pen with no ink". . The application of the unique techniques of brushwork in Chinese painting also has its historical development. Chinese brush ink first experienced a process of first re-using a pen and then using ink. Technically speaking, pen and ink contain a kind of restrained power. The ancients said: "The horizontal line is like a cloud of miles, and the vertical line is like a long lived rattan, such as a mountain falling stone." Through the momentum of the pen and ink changes give people a sense of life. It is not only a simple expression technique, but also an external manifestation of cultural accomplishment and spiritual realm. Chinese brush ink is the crystallization of physical and spiritual content. Chinese painting is an 
enrichment of Chinese people's culture and philosophy, which constitutes its unique artistic aesthetic value. This essence is specifically presented through "pen and ink." We can even say that there is no way to talk about Chinese painting without ink and brush. The famous painter Mr. Huang Binhong said: "There are three flavors in the painting, and there is no way for the pen and ink to understand." And Yun: "The good painters are based on the pen and the Jianxun is in ink." We can see from this that the ink and brush constructs the charm of Chinese painting and discards it. The pen and ink are tantamount to abandoning the soul of Chinese painting. Chinese brush and ink has a close relationship with the development of Chinese culture as the essence of Chinese history and culture.

\section{Chinese Brush and Ink and Emotional Performance}

Chinese brush ink as a special medium, itself has a manifestation of the spiritual realm. Chinese brush ink is the unique temperament and character of Chinese culture. The ink of Chinese painting is the spiritual transmission of Confucianism, Buddhism and Taoism, and it contains the essence of traditional Chinese culture. Chinese brush and ink tells about the fundamental basic laws of the confucian yin and yang alternation of the universe, tells the poetic and calm personality of Taoism, and tells the story of Buddhism's greatness and wisdom. More importantly, the writer can use his Chinese brush and ink to place his thoughts and feelings and shape what he thinks. At the same time, he also arouses the viewer's feelings. Therefore, Chinese brush ink is very much concerned with emotional expression. The landscape paintings by the painter Ni Yunlin in the Yuan Dynasty often gave people a comfortable and elegant taste. The scenery of landscapes has a lot of ecstasy in his paintings. In addition, the landscape paintings by the Qing Dynasty painter Badayamaren are mostly wild mountains and ridges. Most of the rocks are bizarre, leaving people with a sense of desolateness as shown in Figure 1. The painter Shi Tao is a swift and painstaking move that conveys to the people. The tumbler of Qi Baishi, a modern painter, is rich in pen and ink, and satirizes corrupt officials.

Individual positioning pattern application. The positioning pattern in the garment is designed according to the specific size of the specific part of the garment. It is designed for different parts, collars, sleeves, front plackets, hem, back, shoulders, waist, buttocks, legs, etc. Form is the most widely used in traditional clothing. The traditional traditional folk costumes used in the men's design work have precisely applied positioning patterns. On the sleeves, use the form of painting to make reasonable typesetting according to the size of the area. At the front hem, use flowers and plants to draw flowers and plants according to the size of the area.



Fig.1 Bada Shanren landscape paintings

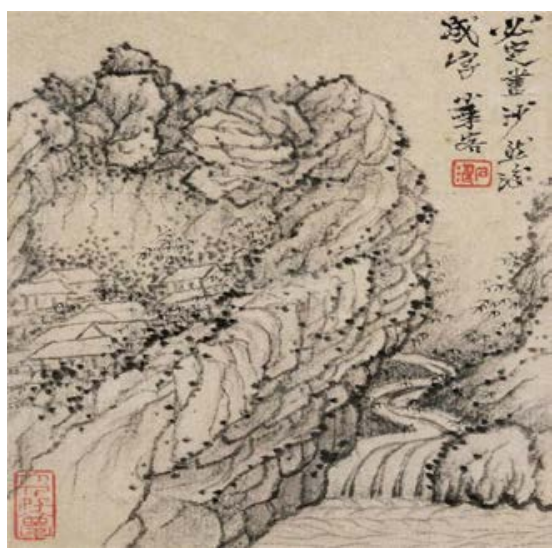

Fig.2 Shi Tao's landscape paintings

Therefore, the painter's painting is not only a copy of the surrounding environment and life, but also a spiritual expression, which is conveyed to the viewer. The artist will integrate his emotions with the scenery. This is where the artistic conception lies. It is precisely because of this artistic conception that the painter paints a perfect work through ink and brush. The artist expresses all his emotional sustenance through writing and ink. All of these perfect works are to convey the painter's 
feelings and ambition through endless changes of ink and brush, leaving the author with unlimited beauty of delusions.

\section{The visual effect of Chinese painting}

Chinese painting pays attention to the purpose of rhyme, and "brush and ink" is the mainstream aesthetic concept. Therefore, Chinese painting stresses the layout of the composition method. The composition method is simply composition, and the artist paints his thoughts and emotions and the artistic laws of the image. This kind of composition is precisely because of the Chinese people's aesthetic habits of thousands of years, and also has its own characteristics and characteristics. When a painter paints, he will show a kind of flexibility, and he will deal with choices in terms of mode of presentation, priority, and cross-reality. Taking nature and the real world as a benchmark, adding their own emotions in space will make the picture more perfect in its concept. In the layout of traditional paintings, the treatment of perspective mainly adopts an unfixed "point of view" to show the vision of the frame. This perspective method is called "moving perspective", also known as "scattering perspective method." In fact, it is more accurate to call the "sports perspective method." Chinese paintings deal with composition with the appreciation and customary requirements of "aspects and scenery" and "moving with people". This will enable the painter to see a comprehensive, far-sighted, and meticulous view, with a visual experience that is hopeful, travelable, and habitable. Therefore, the Chinese painters adopt the view that "the thing is for my own use" that is not a "natural" slave, and boldly and skillfully apply the view method, the Sanyuan method, and the "spectacular perspective" method of "big view and smallness" to organize the images in the frame. Scenery, characters, so as to achieve the artistic effect of the viewers.

Chinese paintings are imaginative and the effect of the picture is ups and downs, which gives viewers a visually appealing impression. Moreover, the picture is smooth, smart, and changing, and the viewer can also see the dynamic beauty of an ethereal change. The so-called "virtual reality, no paintings are in Wonderland," "never breathless, sparsely drivable," these are the best descriptions of Chinese painting. Zhang Yanyuan, the most famous fine art theorist of the Tang Dynasty, once wrote in his "Records of Famous Dynasties": "Far ink and five colors, it is said to be proud. The intention is in the five colors, and the object is like a painting! The appearance of the chapter is very detailed and delicate, and the appearance is meticulous." Thus, the Chinese ink brush focuses on the meaning and purpose. After Zhang Yanyuan's opinion was put forward, this expression of Chinese brush ink gradually became an important art of Chinese painting. Ruler. Chinese brush ink emphasizes the objective existence of beauty, and the existence of beauty lies in the implication of brush and ink art. Expressing the artist's emotions and respecting traditional culture, rather than neglecting traditions and rejecting traditions for their own personality and style. Chinese brush ink is an essential, common, and most essential part of expression. It is a concrete expression of the degree of touch on everyone's emotions. The perfection and expression of the artistic conception of Chinese painting is its highest realm. It is a kind of subjective understanding that the painter obtained after deeply experiencing and experiencing life. Therefore, Chinese painting must reach a realm of "me and me" and advocates "a pen is not a week and a pen is not a work but a compliment." The above characteristics are the rules and characteristics of Chinese brush and ink, and the combination of Chinese ink and ink used in Chinese painting constitute the unique art form of Chinese painting.

\section{Features of Chinese Brush and Ink}

\subsection{Language features.}

Ink painting is the most unique language in Chinese painting. Writing and fortune have their own characteristics. First of all, ink and brush reflect an independent aesthetic value. For example, "If you want to go right and you want to go to the left," you must implement the force you need to carry out the pen and the return. You must never be weak in the middle. "By pen in the pen," it 
reveals a subtle, introverted power. The strokes must be Implicit, avoid stiff. This is a kind of realm and technique that ancient literati and painters need to achieve on ink. It reflects the ancients's aesthetic requirements for handwriting. Second, pen and ink create an organic overall feeling. The Chinese ink paintings are a combination of desires and desires, and they give people a very vivid feeling through the techniques of opposition, reflection, and hope. Third, pen and ink distinguish the relationship between the subject and the guest. Points, blocks, and lines pass through the viewer's consciousness, methodically swaying on rice paper, and the relationship between the subject and the object can be seen through the language of brushstrokes. It is also a demonstration of the Chinese brush and ink aesthetic ability.

\subsection{Charm.}

Qiyun is a special feature that has always permeated the brush ink in China. Chinese painting focuses on "taking breath with a pen and taking rhyme with ink".Sheikh said in the "Six Laws": "The vividness of the rhythm, the use of the bones and the pen, the pictograms of the objects, the distribution of the species, the location of the objects, and the transmission of the writings" have had a profound influence on the Chinese paintings. A major feature of brushstrokes. Through brushwork and composition, it is possible to exhibit a kind of artistic conception. This is difficult for other types of paintings to express. In our traditional brush and ink paintings, we need to give full play to the natural use and flexible changes of brushwork, that is, the highest realm of painting. Forming". In the practice process, many things of the painter are not what they are really pursuing. They ignore the shape and go for pen and ink language. They use spiritual pen and ink to develop their own thoughts and emotions. Brush and ink gradually become their way of expressing emotions. The different brushstrokes used to slow down, relax and ease the emotions are not the same. This is the real realm of Chinese painting.

\subsection{Personality characteristics.}

The Chinese ancients combined the pursuit of traditional painting with the pursuit of philosophical personality. A great artist must have a noble personality and spiritual pursuit. This is the basis of all painting techniques. If there is no such foundation, no matter how good, There is no way to become a master painter. Chinese brush ink embodies the painter's cultivation, mind, talent, character and temperament, and is a true portrayal of personality and spiritual realm. In traditional Chinese culture, many thoughts such as "black and white poles" and "harmony between man and nature" have been used by painters. For example, "Snow Night Map" embodies a kind of thought of doing nothing and governing, Chinese brush ink In the subtle, the Chinese culture has been developed and inherited. This is a feature that no other nation's artistic methods possess.

\section{The Inheritance of Chinese Brush and Ink}

\subsection{Inherit traditions and introduce new ones.}

Chinese brush inks play an important role in the history of Chinese painting. In the process of inheritance, we should follow the principle of "taking its essence and depravity." We must inherit the excellent techniques and methods and remove the dross that does not meet the development of the times. Only by correctly handling the relationship between inheritance and innovation can we give full play to our country's pen and ink painting style. First of all, inheriting the tradition, the development of Chinese brush ink in our country has a history of several thousand years. It can be passed down for a long time, which is enough to prove its artistic charm. Whether it is from the perspective of language features, temperamental characteristics, or personality characteristics, modern artists are worth learning and learning from. Inheriting traditions should begin with Linyi. Through the excellent works of the Linyi ancients, they can appreciate their attitudes and emotions in painting. Wang Yuanzhang once said: "The painting of the heart and the imitating of the ancients must be based on the spirit of the gods and the air and the virtual reality. Reason, well-being. "Note Linyi is often the beginning of the creation, which is the inherent spirit contained in the aesthetic 
object. Secondly, to promote innovation, any kind of art needs to maintain its vitality and artistic power through innovation. The Chinese brush and ink are also the same. We should learn more about the techniques or techniques of foreign painting and connect it with the traditional skills of our country. Art adds new vitality.

\subsection{Break the tradition and advance with the times.}

The development of Chinese brush ink has formed an intrinsic procedure in our country. However, in the development of the times, there are always some traits that are in conflict with the times. As artists who are pursuing freedom, they should try their best to break the inherent mode of this tradition. Combine with the ever-changing era, so as to better help the development of Chinese ink brush. There are many art masters in China who have tried to break through traditional techniques and have made innovations based on the Chinese brush ink. They have achieved very good results. For example, the painter Lee Ke-hsing has had less use of color and created a kind of backlighting effect. On the basis of returning the work to reality, he also exerted the performance of ink and brush; Zhang Daqian turned the ink and brush into a splashing effect, using lines. Scrubbing and brushing depict an unpredictable, blended, and magnificent effect. Since the development of Chinese brush ink, it has long formed a whole, but the two are still two different concepts. The "pen” pursues lines and emphasizes rich changes; "mo" pursues faces, thick, light, and dry. Wetness is both a difference and a unified whole. In the process of future heritage, we can try more possibilities to create better works.

\section{Conclusion}

Nowadays, in the process of inheriting and innovating Chinese paintings, people should pay attention to refusing to engage in extremism and opportunistic inclinations. Chinese paintings have a long history. They embody the ancient people's politics, philosophy, and nature associated with nature, society, and content. The understanding of religion, morality, literature and art is one of the artistic treasures of the Chinese nation. In order to carry forward the traditional form of traditional Chinese painting, and highlight the essence of Chinese painting, people must continue to innovate in the traditional brushwork and concept of Chinese painting in the process of inheritance, integrate the spirit of the times, and enrich the language of painting. In this way, Chinese painting is bound to become more and more Prosperity shines in the world of fine arts.

\section{References}

[1] Tang Xuemei. The application of Chinese brush ink concept in watercolor painting. Hunan Normal University, 2011.

[2] Yu Lin. A brief discussion on the traditional and contemporary characteristics of Chinese brush inks. New West (Theory), 2011.

[3] Yin Guoxia. Research on Chinese brush and ink elements in the design of posters. Journal of Huanghe University of Science and Technology, 2013.

[4] Liu Xiaolong. The brush and ink characteristics of Chinese painting and its contemporary heritage. Art Education Research, 2014. 